\title{
Identification of key genes in calcific aortic valve disease via weighted gene co-expression network analysis
}

\author{
Jin-Yu Sun ${ }^{1 \dagger}$, Yang Hua ${ }^{1 \dagger}$, Hui Shen ${ }^{1}$, Qiang Qu${ }^{1}$, Jun-Yan Kan ${ }^{1}$, Xiang-Qing Kong ${ }^{1}$, Wei Sun ${ }^{1 *}$ and
} Yue-Yun Shen ${ }^{2 *}$

\begin{abstract}
Background: Calcific aortic valve disease (CAVD) is the most common subclass of valve heart disease in the elderly population and a primary cause of aortic valve stenosis. However, the underlying mechanisms remain unclear.

Methods: The gene expression profiles of GSE83453, GSE51472, and GSE12644 were analyzed by 'limma' and 'weighted gene co-expression network analysis (WGCNA)' package in R to identify differentially expressed genes (DEGs) and key modules associated with CAVD, respectively. Then, enrichment analysis was performed based on Gene ontology (GO) and Kyoto Encyclopedia of Genes and Genomes (KEGG) pathway, DisGeNET, and TRRUST database. Protein-protein interaction network was constructed using the overlapped genes of DEGs and key modules, and we identified the top 5 hub genes by mixed character calculation.
\end{abstract}

Results: We identified the blue and yellow modules as the key modules. Enrichment analysis showed that leukocyte migration, extracellular matrix, and extracellular matrix structural constituent were significantly enriched. SPP1, TNC, SCG2, FAM2OA, and CD52 were identified as hub genes, and their expression levels in calcified or normal aortic valve samples were illustrated, respectively.

Conclusions: This study suggested that SPP1, TNC, SCG2, FAM2OA, and CD52 might be hub genes associated with CAVD. Further studies are required to elucidate the underlying mechanisms and provide potential therapeutic targets.

Keywords: Calcific aortic valve disease, Weighted gene co-expression network analysis, Differentially expressed genes, Integrated bioinformatics analysis

\section{Introduction}

Calcific aortic valve disease (CAVD) is the most common subclass of valve heart disease in the elderly population and a primary cause of aortic valve stenosis [1]. It was reported that the incidence of aortic valve stenosis was $2 \%$ in patients $\geq 65$ years old, whereas aortic valve

\footnotetext{
*Correspondence: weisun7919@njmu.edu.cn; lyrmyy@qq.com

†Jin-Yu Sun and Yang Hua contributed equally to this work

${ }^{1}$ Department of Cardiology, The First Affiliated Hospital of Nanjing

Medical University, Nanjing 210000, China

2 Department of Cardiology, Liyang People's Hospital, Liyang 213300, China
}

sclerosis occurred in $26 \%$ of these patients [2, 3]. Considering the prolonged life expectancy and aging of the population, the burden of CAVD is expected to substantially increase from 2.5 million in 2000 to 4.5 million in 2030 [4], thus conferring a high economic and health burden worldwide $[5,6]$.

In CAVD, the fibro-calcific remodeling and pathological thickening of the aortic valve disturb pressure overload and hemodynamic stability. The following progressive cardiac outflow obstruction and left ventricular hypertrophy are likely to result in heart failure and even premature death within a few years $[4,7]$. Accumulating 
studies have revealed that CAVD is a complex multistage disease with sequential and interacting processes, including endothelial dysfunction/injury, lipid deposition, inflammation, extracellular matrix remodeling, dystrophic calcification, and so forth [8-10]. However, mechanisms underlying the development or progression of CAVD remain unclear, and there lacks conservative treatment against CAVD. Surgical or transcatheter aortic valve replacement is the only available treatment, while pharmacotherapy beyond valve replacement is still limited $[5,11]$.

Weighted gene co-expression network analysis (WGCNA) is a bioinformatics algorithm [12], which has been widely applied to explore the changes of transcriptome expression patterns in complex diseases and to identify gene modules associated with clinical features [13-15]. Compared with the standardized analysis of differentially expressed genes (DEGs), WGCNA is a powerful systematic analysis method to recognize the higher-order correlation between genes instead of detecting disease-related individual genes. Our study identified DEGs between calcified and normal aortic valve samples based on the gene expression profiles of GSE83453 and GSE51472, GSE12644. Moreover, WGCNA was performed on the GSE83453 dataset to screen key genes and modules related to CAVD. Then, the enrichment analysis of genes in key modules was used to explore the molecular mechanisms underlying CAVD. Finally, we identified hub genes related to CAVD and establish a protein-protein interaction (PPI) network.

\section{Materials and methods \\ Microarray data}

The gene expression profiles of GSE83453 [16], GSE51472 [17], and GSE12644 [18] were acquired from the Gene Expression Omnibus database. All three gene expression profiles were acquired from human samples. Dataset GSE83453 was performed based on the platform GPL10558 (Illumina HumanHT-12 V4.0 expression beadchip), which includes 9 calcified aortic valve samples and 8 normal aortic valve samples [16]. Series GSE51472 was performed by GPL570 (Affymetrix Human Genome U133 Plus 2.0 Array) and included 5 calcified aortic valve samples and 5 normal aortic valve samples [17]. GSE12644, based on the GPL570 platform, included 10 calcified and 10 normal aortic valve samples (Fig. 1).

\section{Data pre-processing and DEG screening}

We performed $\log _{2}$-transformation, background correction, and quantile normalization on the expression profiles of GSE83453, GSE51472, and GSE12644 using the 'linear models for microarray data (limma)' package in $\mathrm{R}$ 3.6.1 software (R Foundation for Statistical Computing,
Vienna, Austria). The probe IDs were converted into gene symbols according to the annotation file. For multiple probes mapping to a single gene, the average expression value of all its corresponding probes was used. Importantly, the batch effect was corrected using the combat function of the 'SVA' package, which is a widely used empirical Bayes method for batch correction [19]. To control the false discovery rate caused by multiple testing, the adjusted $P$-value was applied. After pre-processing, DEGs between calcified and normal aortic valve samples were screened with a threshold of adjusted $P$-value $<0.05$ and $\mid \log _{2}$ fold-change (FC) $\mid \geq 0.5$ by 'limma' package. Furthermore, the DEGs were visualized as a volcano plot and heatmap using 'ggplot2' and 'pheatmap' package.

\section{Weighted gene co-expression network analysis (WGCNA)} WGCNA is an algorithm for constructing a co-expression network, which reveals the correlation patterns across genes and provides the biologically functional interpretations of network modules. As previously described [20], we selected the top $25 \%$ most variant genes in GSE83453 to construct a co-expression network using the 'WGCNA' package (version 1.60). After evaluating the presence of obvious outliers by cluster analysis, the one-step network construction function was used to construct the co-expression network and identify key modules. Moreover, to identify the significance of each module, we summarized the module eigengene (ME) based on the first principal component of the module expression, and the module-trait relationships were assessed according to the correlation between MEs and clinical traits. Then, we evaluated the correlation strength by module significance (MS), referring to the average absolute gene significance (GS) of all genes within one module. Notably, the GS value was determined by the $\log _{10}$-transformation of the $P$-value in the linear regression between expression and clinical traits. In general, the modules with the highest MS values were considered as the key modules.

Furthermore, we used the modulePreservation function to evaluate the preservation levels of key modules. $\mathrm{Z}_{\text {summary }}$ analysis combines different preservation statistics into one single overall measure of preservation. According to WGCNA instruction, a higher $Z_{\text {summary }}$ value indicated the stronger the evidence that a module should be preserved: the module with $Z_{\text {summary }}$ value $<2$ indicated 'no evidence', $2<Z_{\text {summary }}$ value $<10$ indicated 'weak evidence', and $Z_{\text {summary }}$ value $>10$ indicated strong evidence. Additionally, to confirm the clustering ability of the key modules, we also performed principal component analysis (PCA) on the gene expression profile of the key modules. 


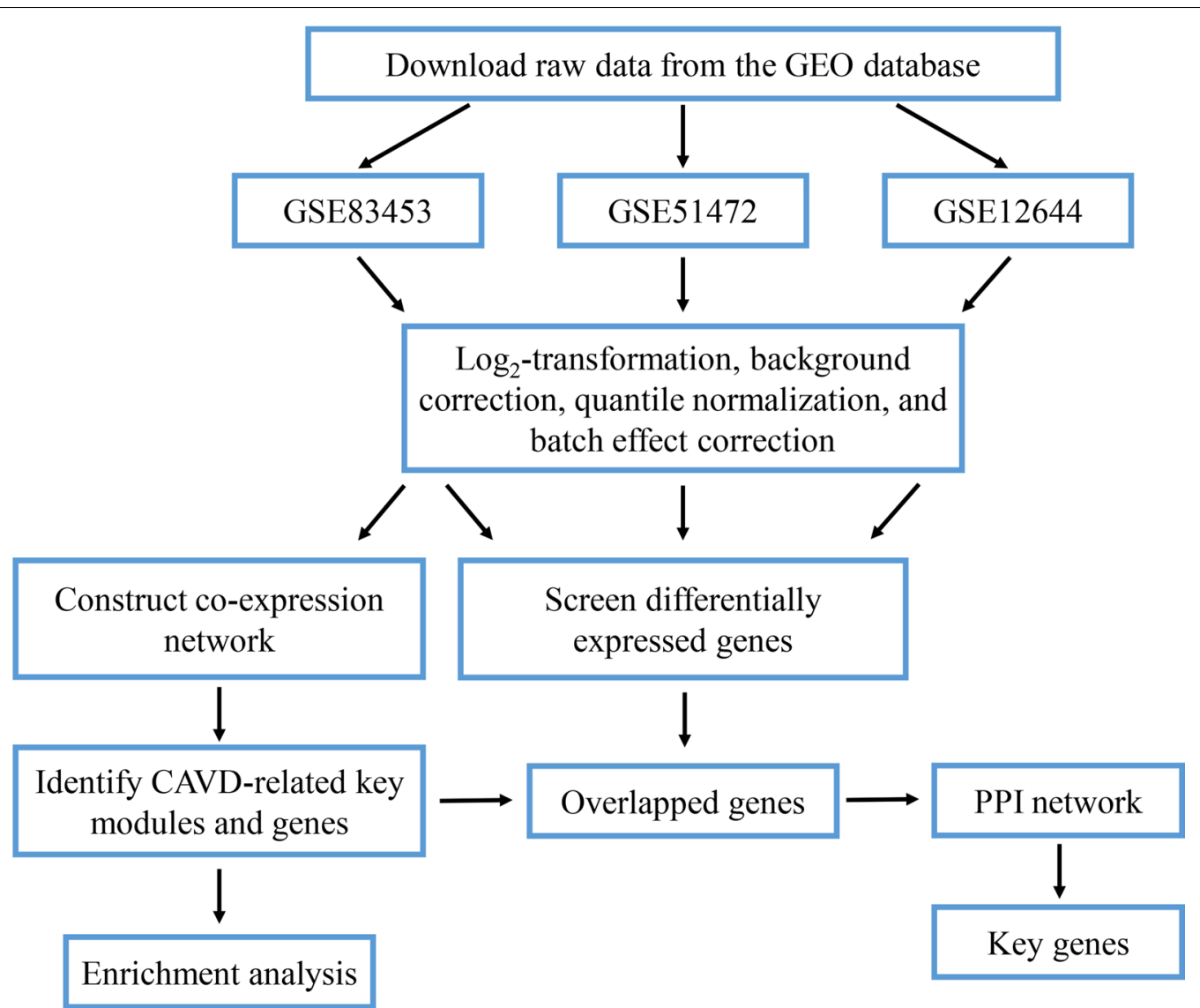

Fig. 1 Flow chart of data preparation, processing, and analysis. GEO Gene Expression Omnibus, CAVD calcific aortic valve disease, PPI proteinprotein interaction

\section{Enrichment analysis of genes in key modules}

To understand the biological meaning of the genes in key modules, we used the 'clusterProfiler' package to perform Gene Ontology (GO) and Kyoto Encyclopedia of Genes and Genomes (KEGG) pathway enrichment analysis. Moreover, pathway and process enrichment analysis were also conducted and visualized by Metascape [21], a biologist-oriented tool for analyzing systems-level datasets. To illustrate the relationships between the terms, a subset of terms was further selected and rendered as a network plot (with a similarity of $>0.3$ ). Each node represented an enriched term and colored first by its cluster ID. Furthermore, we performed association and enrichment analysis using DisGeNET [22] and TRRUST [23] database and visualized by 'ggplot2' package. DisGeNET is a database of gene-disease associations, which collects one of the largest publicly available collections of genes and human diseases-related variants [22]. TRRUST is a manually-curated database of transcriptional regulatory networks based on a sentence-based text mining approach, containing 8444 and 6552 transcription factor-target regulatory relationships of 800 human and 828 mouse transcription factors, respectively [23].

\section{PPI network construction and identification of hub genes}

In WGCNA, we defined the genes from key modules with $\mid$ module membership (MM) $\mid \geq 0.8$ and $|G S| \geq 0.2$ as key genes. After overlapping the DEGs and key genes from WGCNA, we inputted these genes into the STRING (http://string-db.org) database to collect the interaction of target proteins with a medium confidence score of $>0.4$ [24] and constructed a PPI network by Cytoscape software (v3.7.2) [25]. Further, we identified the top 5 hub genes using the Cytoscape plug-in software 'cytoHubba' based on mixed character calculation.

\section{The expression of hub genes in calcified or normal aortic valve sample}

We compared the expression of hub genes between calcified or normal aortic valves from GSE83453 by Student's t-test. The 'ggplot2' and 'ggsignif' packages were used to create the box plots of the expression of hub genes. 


\section{Results}

DEGs between calcified and normal aortic valve

After data pre-processing of the dataset GSE83453, GSE51472, and GSE1264, we screened DEGs according to the cut-off criterion of adjusted $P$-value $<0.05$ and $\left|\log _{2} \mathrm{FC}\right| \geq 0.5$. In GSE83453, we identified 269 up-regulated and 170 down-regulated genes. The volcanic diagram for all genes and the expression heatmap of the top 10 DEGs are shown in Fig. 2a, b. In GSE51472, 963 genes were up-regulated, and 978 genes were down-regulated
(Fig. 2c, d). A total of 28 up-regulated and 11 down-regulated genes were identified in GSE12644 (Fig. 2e, f).

\section{Construction of co-expression network and key modules identification}

With a scale-free network and topological overlaps, a hierarchical clustering tree was created based on the dynamic hybrid cut (Fig. 3a). Based on the scale-free topology criterion, the soft-thresholding power of 12 was selected (scale-free $R^{2}=0.88$; Fig. $3 b, c$ ), and a total of 24

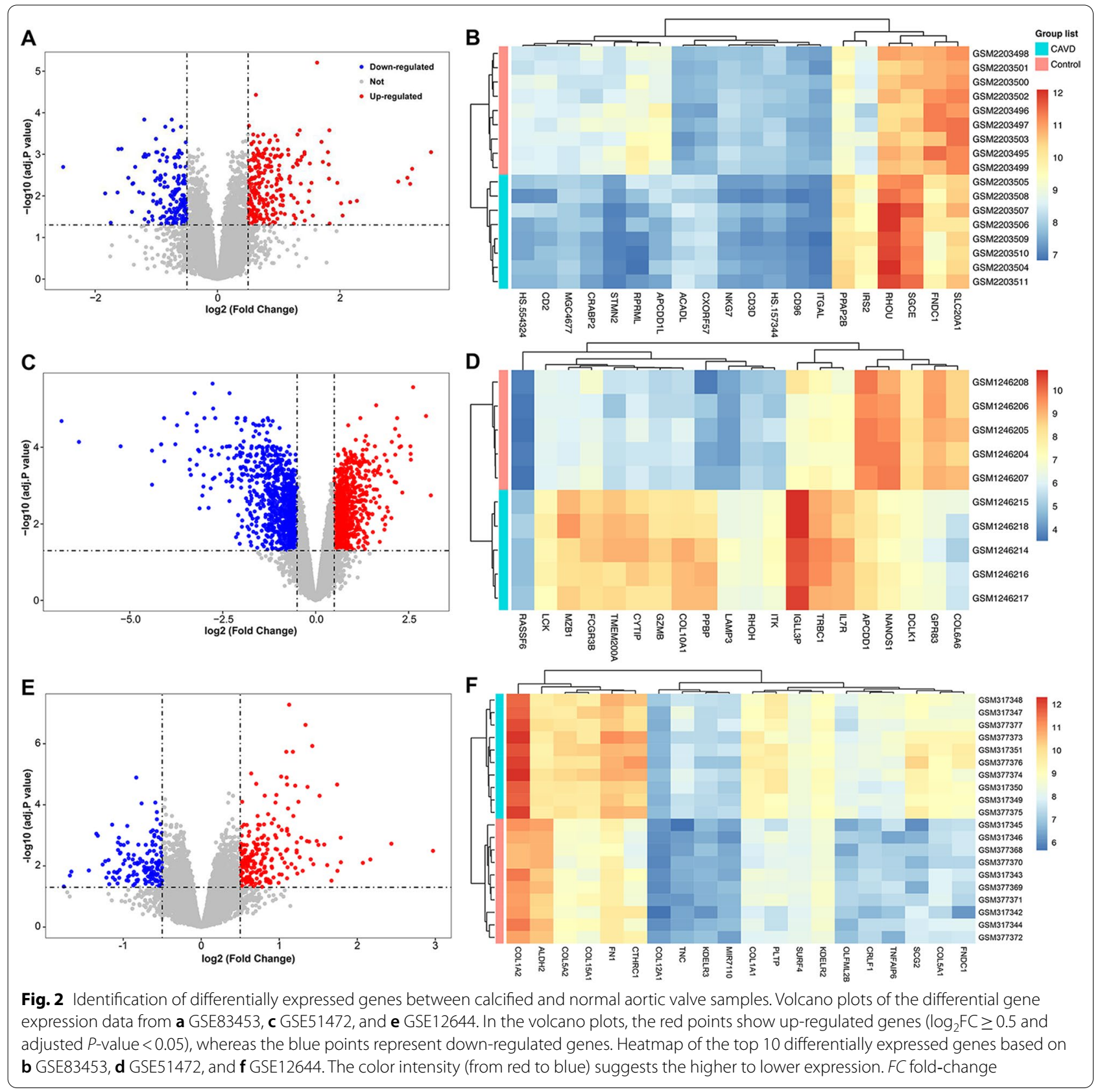




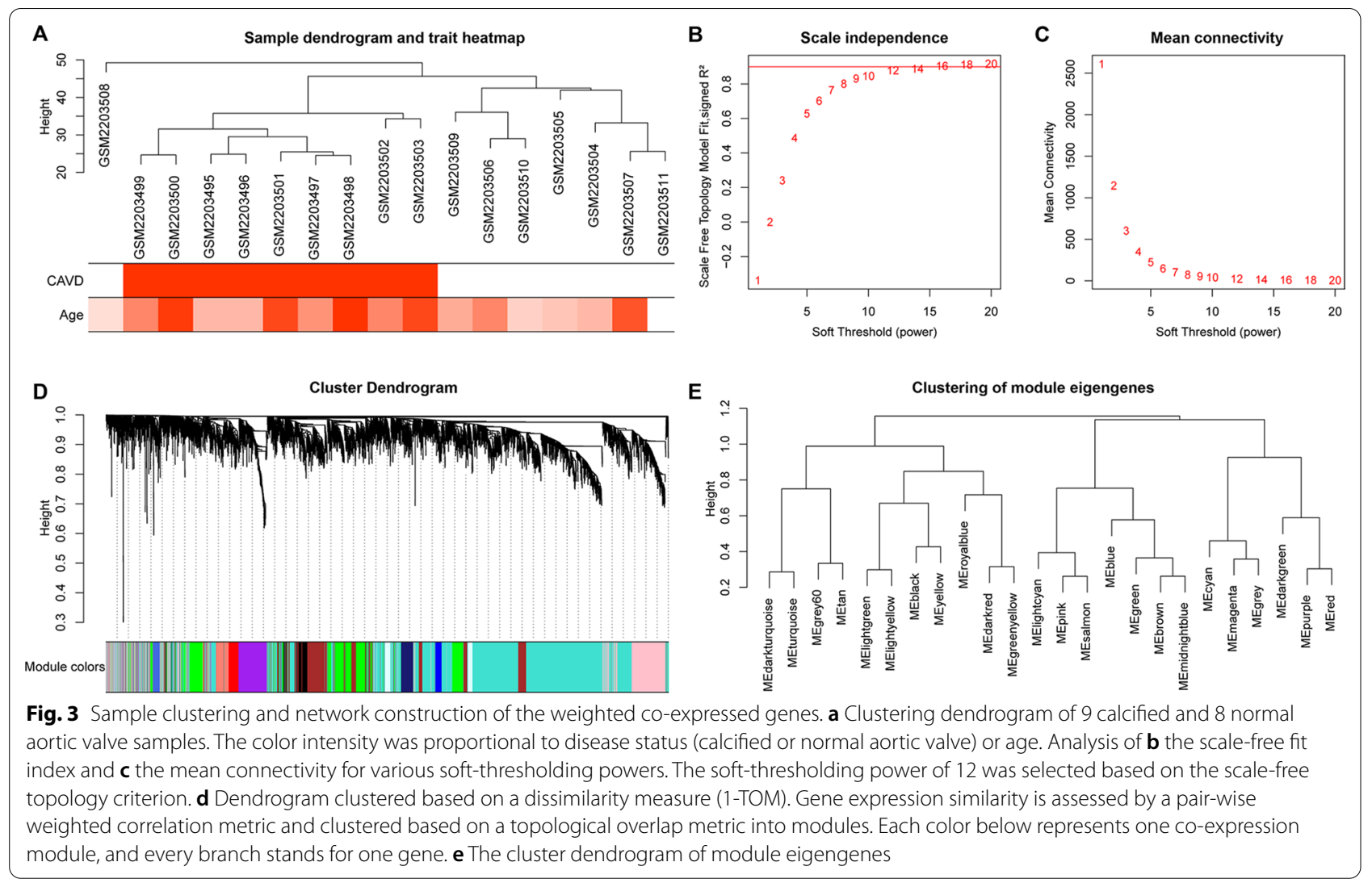

modules were identified for further analysis. In addition, the gray module included all genes that could not be put into any other modules. The cluster dendrogram of the modules is shown in Fig. 3d, whereas the clustering of module eigengenes is provided in Fig. 3e.

Moreover, we analyzed the association of gene modules with CAVD status and age (Fig. 4a). The blue module showed the highest positive correlation $(r=0.93$, $P=5 \mathrm{e}-08$ ), while the yellow module was the most negatively associated with CAVD $(r=-0.83, P=3 e-05)$. As shown in Fig. $4 \mathrm{~b}$, the blue and yellow modules were also the two most significantly correlated with CAVD. Therefore, we identified the blue and yellow modules as key modules for further analysis. A total of 436 and 82 genes were included in the blue and yellow modules, respectively. Additionally, we illustrated the correlation between module membership and GS for CAVD in blue (correlation coefficient $=0.91, \mathrm{P}<1 \mathrm{e}-200$ ) and yellow module (correlation coefficient $=0.74, \mathrm{P}=1.8 \mathrm{e}-111$ ), respectively (Fig. 4c, d).

Figure $4 \mathrm{e}$ shows the module preservation statistics, and the $Z_{\text {summary }}$ values of both blue and yellow modules were $>10$. The PCA on the genes in blue and yellow modules illustrated the overlap of samples within the CVAD or control groups, which suggested the highlevel clustering ability of the key modules (Fig. 4f).

\section{Enrichment analysis of key modules}

We performed functional enrichment analysis on the blue and yellow modules based on GO and KEGG databases. As shown in Fig. 5a, the ontology encompasses three domains (biological process, cellular component, and molecular function). The enriched biological processes were mainly involved in leukocyte migration, extracellular structure organization, extracellular matrix organization, $\mathrm{T}$ cell activation, cell-substrate adhesion, and renal system development. The cellular components were mainly enriched in the extracellular matrix and collagen-containing extracellular matrix, whereas the enriched molecular functions were mainly involved in extracellular matrix structural constituent. In Fig. 5b, KEGG pathway analysis shows that the human papillomavirus infection and PI3K-Akt signaling pathway were the most enriched pathways, followed by focal adhesion, extracellular matrix-receptor interaction, cell adhesion molecules, and pathogenic Escherichia coli infection. In addition, Fig. 5c shows the top 20 clusters of the pathway and process enrichment 


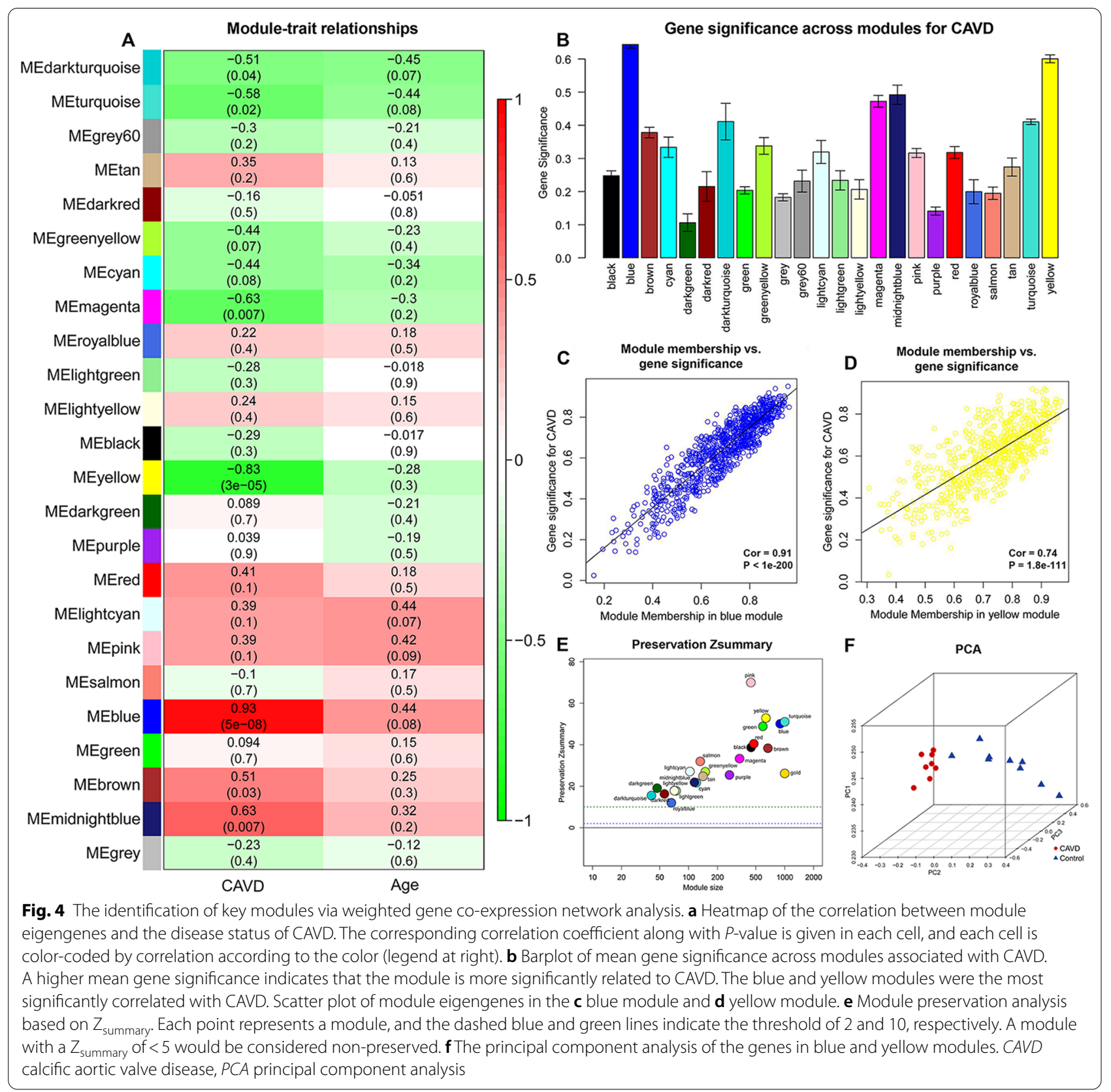

analysis, such as regulation of cell adhesion, regulation of cell projection organization, ossification, collagen metabolic process, and so forth.

Moreover, the enrichment analysis in DisGeNET showed that the genes in key modules were significantly associated with liver cirrhosis, immunologic deficiency syndromes, and pulmonary fibrosis (Fig. 5d). Based on the TRRUST database, we also identified multiple transcription factors associated with the genes in key modules, including SP1, FOS, JUN, ETS1, NFKB1, and RFX5 (Fig. 5e).
PPI network construction and identification of hub genes With a threshold of $|\mathrm{MM}| \geq 0.8$ and $|\mathrm{GS}| \geq 0.2,252$ and 210 key genes were identified from the blue and yellow modules, respectively. After overlapping the DEGs and key genes, a total of 55 genes were screened out (Fig. 6a; Additional file 1: Table 1). The PPI network of these genes is shown in Fig. 6b. Moreover, CytoHubba revealed the top 5 hub genes according to the topological parameters of the interaction network, including secreted phosphoprotein 1 (SPP1), tenascin C (TNC), secretogranin II (SCG2), family with sequence similarity 20-member A 


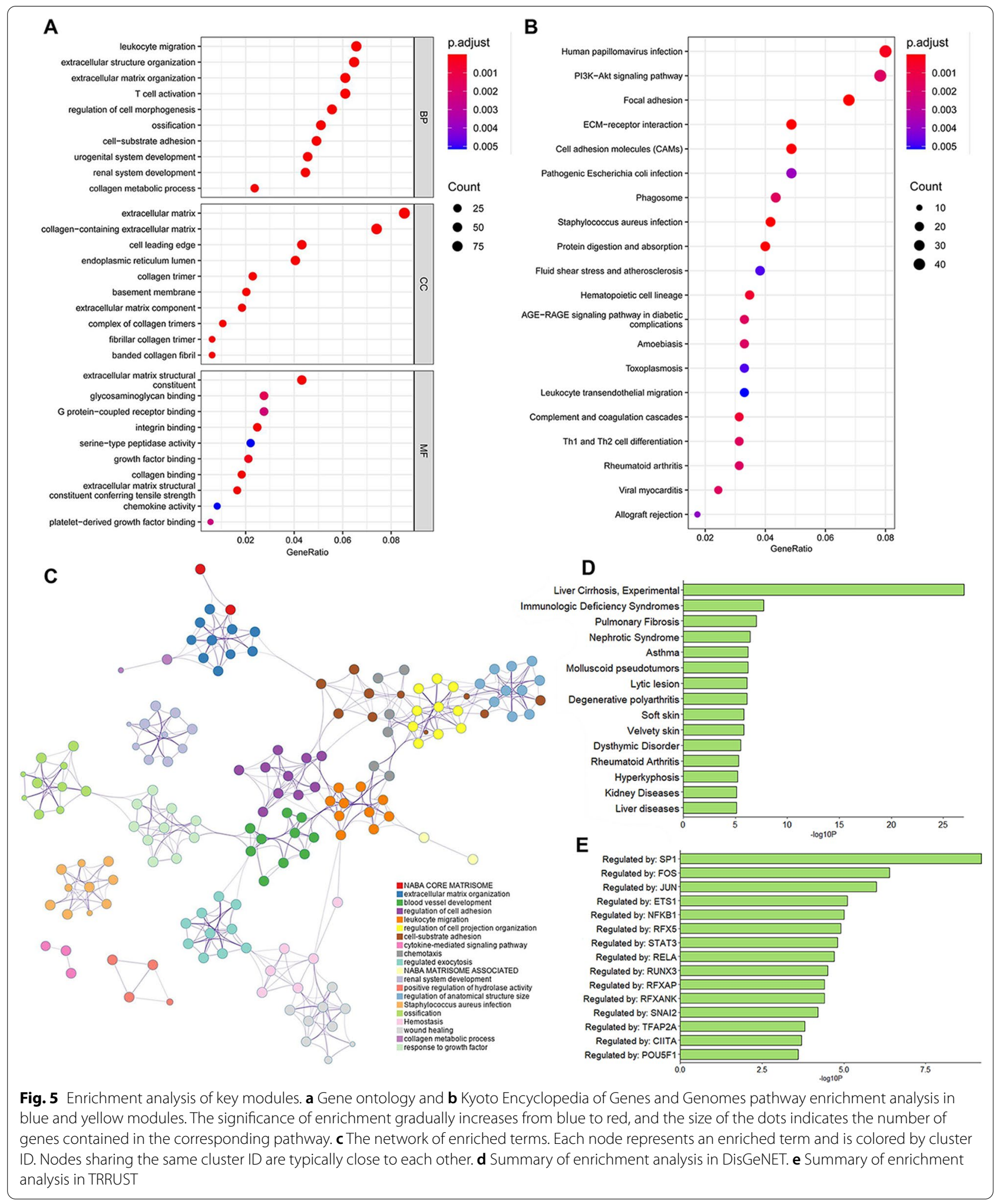




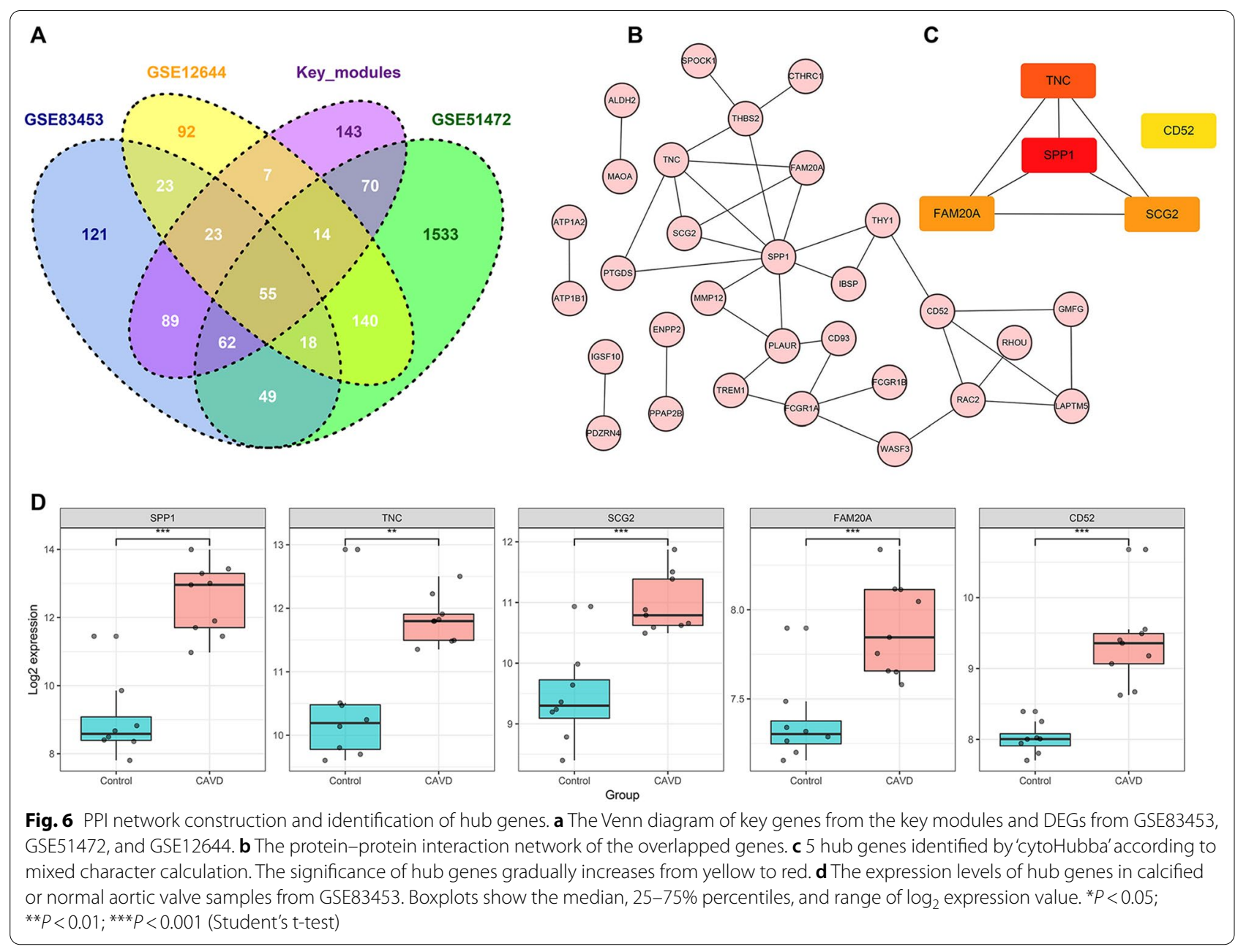

(FAM20A), and CD52 (Fig. 6c). As shown in Fig. 6d, the expression of SPP1, TNC, SCG2, FAM20A, and CD52 was all significantly up-regulated in calcific aortic valve disease.

\section{Discussion}

Compared with previous studies[26, 27], we used three gene expression profiles from 24 human calcified aortic valve samples and 23 normal samples, and both expression analysis and weighted co-expression network analysis were applied to identify key genes related to CAVD comprehensively. Our study performed WGCNA to reveal the correlation patterns between genes across samples and interpret the straightforward biological function of gene network modules. We also used multiple databases in the enrichment analysis to explore the possible mechanisms underlying CAVD, including GO, KEGG, DisGeNET, and TRRUST databases. Notably, the batch effect was corrected using an empirical Bayes framework in our study.
CAVD is a chronic inflammatory disease sharing similar pathogenesis with atherosclerosis [9]. The sterile inflammation in all stages of CAVD is accompanied by extensive immune cell infiltration (such as macrophages, T cells, and mast cells) [28-31]. In this process, cell adhesion molecules facilitate their adhesion and transendothelial migration via interacting with the ligands on the immune cells $[32,33]$. The GO analysis revealed that leukocyte migration, extracellular structure organization, extracellular matrix organization, $\mathrm{T}$ cell activation, and cell-substrate adhesion were significantly enriched. These invading immune cells release pro-fibrotic and pro-inflammatory cytokines, contributing to the reactive oxygen species production, mast cell degranulation, and synthesis of valvular extracellular matrix[34], thus ultimately increasing value thickness. Among the many cytokines, TNF- $\alpha$ has been demonstrated as a particularly important regulator in CAVD that triggers the transition of valvular smooth muscle cells and myofibroblasts to myofibroblasts via activating Wnt pathway activation 
and increasing ALP, BMP-2, and RUNX2 expression [35-37]. However, the role of other pro-fibrotic and proinflammatory cytokines remains limited.

TNC, also named Tenascin-C, is a large extracellular matrix glycoprotein and the main component of extracellular matrix-induced dynamic mechanical stress [38]. Our study showed that TNC was significantly up-regulated in calcified aortic valves. TNC is transiently present during extracellular matrix remodeling and is involved in cell differentiation, proliferation, and migration [39]. In healthy valves, TNC was primarily distributed on the basement membrane beneath the endothelial cells, while stenotic valves exhibited no such distribution but immunoreactivity in the deeper layers, especially in calcific nodules [40]. Interestingly, TNC has been demonstrated to induce phenotypic changes of fibroblasts to myofibroblasts in human breast cancer. Moreover, TNC is also reported to be detrimental in cardiac fibrotic remodeling and hypertrophy after myocardial infarction, probably via up-regulating angiotensin-converting enzyme [41, 42].

In CAVD, a disorganized and disrupted valvular extracellular matrix is susceptible to the dystrophic calcification induced by calcium and phosphate deposits. Activated valve interstitial cells may differentiate into myofibroblasts and osteoblasts, contributing to the fibrosis and ossification of the aortic valve, respectively [43, 44]. SPP1 (also termed osteopontin) plays a central role in the progression of biomineralization, which accelerates reparative fibrosis and contributes to the differentiation of valvular interstitial cells into osteoblasts [45-47]. Consistent with our research, multiple studies have revealed an elevated SPP1 expression in calcified aortic valves compared with healthy valves [48, 49]. Vadana et al. [50] analyzed the high glucose-induced calcification on gelatin-populated 3D constructs with human valvular endothelial cells and interstitial cells. The results showed increased calcium deposits and up-regulated osteogenic molecules, including SPP1, osteocalcin, and bone morphogenetic protein [50]. In previous bioinformatic research on 6 valvular tissue samples from patients with aortic stenosis, RNA-Seq analysis suggested that SPP1 was significantly up-regulated in aortic valve sclerosis [51]. Moreover, from the perspective of immune cell infiltration and cytokine response, macrophage-derived valvular extracellular matrix protein SPP1 could contribute to immune cell migration, cytokine release, as well as calcium deposition [49].

SCG2, or secretogranin II, is a 587 amino acid long protein from the chromogranin-secretogranin protein family [52]. We found that SCG2 was significantly up-regulated in calcific valves and was predicted to be a CAVD-related gene by WGCNA. Secretoneurin is an active neuropeptide derived from secretogranin II, an abundant protein in neuroendocrine storage vesicles [53]. Secretoneurin is a key modulator in the inflammatory process [54], including stimulating monocyte chemotaxis [55], increasing the permeability through monolayered coronary endothelial cells [56], and transendothelial transport of cells and substances [57]. Secretogranin has been demonstrated to act as a pro-angiogenic agent and induce postnatal vasculogenesis by enhancing VEGF signaling in endothelial cells [58], promoting inflammatory cell/macrophage accumulation, and stimulating VSMC proliferation [59]. As angiogenesis [60] and inflammation [8] may contribute to CAVD progression, it is necessary to ascertain the exact role of SCG2 in CAVD.

Our study showed that FAM20A was significantly upregulated in calcified aortic valves, which might contribute to valve calcification progression. The Fam 20 families (including FAM2OA, FAM2OB, and FAM20C) are novel kinases phosphorylating secreted proteins and proteoglycans. FAM20A activates Fam20C, which phosphorylates hundreds of secreted proteins, whereas Fam20B could regulate proteoglycan synthesis via phosphorylating a xylose residue [61, 62]. Mutations in FAM20A have been demonstrated to cause amelogenesis imperfecta and enamel renal syndrome [63, 64]. However, the role of FAM2OA in aortic valve calcification remains unclear.

CD52, also known as CAMPATH-1 antigen, is a glycoprotein of 12 amino acids anchored to glycosylphosphatidylinositol [65]. In our research, CD52 expression was significantly up-regulated in calcific valves compared with normal samples. As a nonmodulating membrane glycoprotein, it is highly expressed on the surface of immune cells (e.g., mature lymphocytes, especially memory $\mathrm{CD} 4^{+} \mathrm{T}$ cells and $\mathrm{B}$ cells, monocytes, and dendritic cells) [66]. It has been shown that CD52 may provide costimulatory signals for T-cell activation and proliferation [65]. However, Rashidi et al. [67] reported another formation of CD52, soluble CD52, which was associated with suppressing inflammatory cytokine production by limiting TLR suppress-induced NF- $\kappa B$ activation in the innate immune cell, indicating its anti-inflammatory function and immunotherapeutic agent.

Several limitations of the study should be mentioned. First, the paucity of confirmatory experiments is a significant limitation. Considering the accumulating publicly available disease-related gene expression profiles, it would be necessary to perform a comprehensive analysis and make full use of the existing resources economically and effectively. However, a further mechanism investigation based on the current dataset would be unreliable. Therefore, this study is descriptive without mechanism research, and further molecular biology experiments would be necessary to validate the results of the present study. Second, all samples were derived from male 
individuals. Given the sex-dependent features of CAVD [68], the sex-unbalanced samples are likely to result in selection bias. Third, although a total of 47 samples were used for analysis, the input data might still be insufficient to identify and validate key genes in the CAVD development.

\section{Conclusions}

This study suggested that SPP1, TNC, SCG2, FAM2OA, and CD52 might be key genes associated with the development and progression of CAVD. Further studies are required to elucidate the underlying mechanisms and provide possible therapeutic targets.

\begin{abstract}
Abbreviations
CAVD: Calcific aortic valve disease; WGCNA: Weighted gene co-expression network analysis; DEG: Differentially expressed gene; GO: Gene ontology; KEGG: Kyoto Encyclopedia of Genes and Genomes; PPI: Protein-protein interaction; ME: Module eigengene; MS: Module significance; GS: Gene significance; PCA: Principal component analysis.
\end{abstract}

\section{Supplementary Information}

The online version contains supplementary material available at https://doi. org/10.1186/s12920-021-00989-w.

Additional file $\mathbf{1 . 5 5}$ overlapped genes and their corresponding module color.

\section{Acknowledgements}

Mr. Jin-Yu Sun congratulated Dr. Shan-Shan Li on her graduation. Wish her a bright future.

\section{Authors' contributions}

Conceptualized and designed the study: JYS, YH, WS and YYS; Data analysis: JYS, YH and HS; Result interpretation: JYS, QQ and JYK; Wrote the paper: JYS and XQK. All authors read and approved the final manuscript.

\section{Funding}

None.

\section{Availability of data and materials}

All the data were acquired from the Gene Expression Omnibus (GEO) database.

\section{Declarations}

Ethics approval and consent to participate

Not applicable.

\section{Consent for publication}

Not applicable.

\section{Competing interests}

The authors declare that they have no competing interests.

Received: 5 February 2021 Accepted: 17 May 2021

Published online: 21 May 2021

\section{References}

1. lung B, Vahanian A. Epidemiology of acquired valvular heart disease. Can J Cardiol. 2014;30(9):962-70.

2. Stewart BF, Siscovick D, Lind BK, Gardin JM, Gottdiener JS, Smith VE, et al. Clinical factors associated with calcific aortic valve disease. Cardiovascular Health Study. J Am Coll Cardiol. 1997;29(3):630-4.

3. Vahanian A, Alfieri O, Andreotti F, Antunes MJ, Baron-Esquivias G, Baumgartner $\mathrm{H}$, et al. Guidelines on the management of valvular heart disease (version 2012): the Joint Task Force on the Management of Valvular Heart Disease of the European Society of Cardiology (ESC) and the European Association for Cardio-Thoracic Surgery (EACTS). Eur J Cardiothorac Surg. 2012:42(4):S1-44.

4. Yutzey KE, Demer LL, Body SC, Huggins GS, Towler DA, Giachelli CM, et al. Calcific aortic valve disease: a consensus summary from the Alliance of Investigators on Calcific Aortic Valve Disease. Arterioscler Thromb Vasc Biol. 2014;34(11):2387-93.

5. Nishimura RA, Otto CM, Bonow RO, Carabello BA, Erwin JP 3rd, Fleisher LA, et al. 2017 AHA/ACC focused update of the 2014 AHA/ACC guideline for the management of patients with valvular heart disease: a report of the American College of Cardiology/American Heart Association Task Force on Clinical Practice Guidelines. J Am Coll Cardiol. 2017;70(2):252-89.

6. Thaden JJ, Nkomo VT, Enriquez-Sarano M. The global burden of aortic stenosis. Prog Cardiovasc Dis. 2014;56(6):565-71.

7. Lindman BR, Clavel MA, Mathieu P, lung B, Lancellotti P, Otto CM, et al. Calcific aortic stenosis. Nat Rev Dis Primers. 2016;3(2):16006.

8. Mathieu P, Boulanger MC. Basic mechanisms of calcific aortic valve disease. Can J Cardiol. 2014;30(9):982-93.

9. Kostyunin AE, Yuzhalin AE, Ovcharenko EA, Kutikhin AG. Development of calcific aortic valve disease: do we know enough for new clinical trials? J Mol Cell Cardiol. 2019;132:189-209.

10. Towler DA. Molecular and cellular aspects of calcific aortic valve disease. Circ Res. 2013;113(2):198-208.

11. Nishimura RA, Otto CM, Bonow RO, Carabello BA, Erwin JP 3rd, Guyton RA, et al. 2014 AHA/ACC guideline for the management of patients with valvular heart disease: executive summary: a report of the American College of Cardiology/American Heart Association Task Force on Practice Guidelines. J Am Coll Cardiol. 2014;63(22):2438-88.

12. Langfelder P, Horvath S. WGCNA: an R package for weighted correlation network analysis. BMC Bioinform. 2008;29(9):559.

13. Wittenberg GM, Greene J, Vertes PE, Drevets WC, Bullmore ET. Major depressive disorder is associated with differential expression of innate immune and neutrophil-related gene networks in peripheral blood: a quantitative review of whole-genome transcriptional data from casecontrol studies. Biol Psychiatry. 2020;88:625.

14. Furusawa $\mathrm{H}$, Cardwell JH, Okamoto T, Walts AD, Konigsberg IR, Kurche JS, et al. Chronic hypersensitivity pneumonitis, an interstitial lung disease with distinct molecular signatures. Am J Respir Crit Care Med. 2020;202:1430-44.

15. Wang G, Yu J, Yang Y, Liu X, Zhao X, Guo X, et al. Whole-transcriptome sequencing uncovers core regulatory modules and gene signatures of human fetal growth restriction. Clin Transl Med. 2020;9(1):9.

16. Guauque-Olarte S, Droit A, Tremblay-Marchand J, Gaudreault N, Kalavrouziotis D, Dagenais F, et al. RNA expression profile of calcified bicuspid, tricuspid, and normal human aortic valves by RNA sequencing. Physiol Genomics. 2016:48(10):749-61.

17. Ohukainen P, Syvaranta S, Napankangas J, Rajamaki K, Taskinen P, Peltonen T, et al. MicroRNA-125b and chemokine CCL4 expression are associated with calcific aortic valve disease. Ann Med. 2015;47(5):423-9.

18. Bosse Y, Miqdad A, Fournier D, Pepin A, Pibarot P, Mathieu P. Refining molecular pathways leading to calcific aortic valve stenosis by studying gene expression profile of normal and calcified stenotic human aortic valves. Circ Cardiovasc Genet. 2009;2(5):489-98.

19. Leek JT, Johnson WE, Parker HS, Jaffe AE, Storey JD. The sva package for removing batch effects and other unwanted variation in high-throughput experiments. Bioinformatics. 2012;28(6):882-3.

20. Yuan Y, Chen J, Wang J, Xu M, Zhang Y, Sun P, et al. Identification hub genes in colorectal cancer by integrating weighted gene co-expression network analysis and clinical validation in vivo and vitro. Front Oncol. 2020;10:638. 
21. Zhou Y, Zhou B, Pache L, Chang M, Khodabakhshi AH, Tanaseichuk O, et al. Metascape provides a biologist-oriented resource for the analysis of systems-level datasets. Nat Commun. 2019;10(1):1523.

22. Pinero J, Bravo A, Queralt-Rosinach N, Gutierrez-Sacristan A, Deu-Pons J, Centeno $\mathrm{E}$, et al. DisGeNET: a comprehensive platform integrating information on human disease-associated genes and variants. Nucleic Acids Res. 2017;45(D1):D833-9.

23. Han H, Cho JW, Lee S, Yun A, Kim H, Bae D, et al. TRRUST v2: an expanded reference database of human and mouse transcriptional regulatory interactions. Nucleic Acids Res. 2018;46(D1):D380-6.

24. Szklarczyk D, Gable AL, Lyon D, Junge A, Wyder S, Huerta-Cepas J, et al. STRING v11: protein-protein association networks with increased coverage, supporting functional discovery in genome-wide experimental datasets. Nucleic Acids Res. 2019;47(D1):D607-13.

25. Shannon P, Markiel A, Ozier O, Baliga NS, Wang JT, Ramage D, et al. Cytoscape: a software environment for integrated models of biomolecular interaction networks. Genome Res. 2003;13(11):2498-504.

26. Teng $\mathrm{P}, \mathrm{Xu} X, \mathrm{Ni}$ C, Yan H, Sun Q, Zhang E, et al. Identification of key genes in calcific aortic valve disease by integrated bioinformatics analysis. Medicine (Baltimore). 2020;99(29):e21286.

27. Zhang Y, Ma L. Identification of key genes and pathways in calcific aortic valve disease by bioinformatics analysis. J Thorac Dis. 2019;11(12):5417-26

28. Edep ME, Shirani J, Wolf P, Brown DL. Matrix metalloproteinase expression in nonrheumatic aortic stenosis. Cardiovasc Pathol. 2000;9(5):281-6.

29. Steiner I, Stejskal V, Zacek P. Mast cells in calcific aortic stenosis. Pathol Res Pract. 2018;214(1):163-8.

30. Kaden JJ, Dempfle CE, Grobholz R, Tran HT, Kilic R, Sarikoc A, et al. Interleukin-1 beta promotes matrix metalloproteinase expression and cell proliferation in calcific aortic valve stenosis. Atherosclerosis 2003;170(2):205-11.

31. Olsson M, Dalsgaard CJ, Haegerstrand A, Rosenqvist M, Ryden L, Nilsson J. Accumulation of T lymphocytes and expression of interleukin-2 receptors in nonrheumatic stenotic aortic valves. J Am Coll Cardiol. 1994:23(5):1162-70.

32. Muller WA. Mechanisms of transendothelial migration of leukocytes. Circ Res. 2009;105(3):223-30

33. Ghaisas NK, Foley JB, O'Briain DS, Crean P, Kelleher D, Walsh M. Adhesion molecules in nonrheumatic aortic valve disease: endothelial expression, serum levels and effects of valve replacement. J Am Coll Cardiol. 2000;36(7):2257-62

34. Michaud M, Balardy L, Moulis G, Gaudin C, Peyrot C, Vellas B, et al. Proinflammatory cytokines, aging, and age-related diseases. J Am Med Dir Assoc. 2013;14(12):877-82.

35. Al-Aly Z, Shao JS, Lai CF, Huang E, Cai J, Behrmann A, et al. Aortic Msx2Wnt calcification cascade is regulated by TNF-alpha-dependent signals in diabetic Ldlr-/- mice. Arterioscler Thromb Vasc Biol. 2007;27(12):2589-96.

36. Kaden JJ, Kilic R, Sarikoc A, Hagl S, Lang S, Hoffmann U, et al. Tumor necrosis factor alpha promotes an osteoblast-like phenotype in human aortic valve myofibroblasts: a potential regulatory mechanism of valvular calcification. Int J Mol Med. 2005;16(5):869-72.

37. Yu Z, Seya K, Daitoku K, Motomura S, Fukuda I, Furukawa K. Tumor necrosis factor-alpha accelerates the calcification of human aortic valve interstitial cells obtained from patients with calcific aortic valve stenosis via the BMP2-Dlx5 pathway. J Pharmacol Exp Ther. 2011;337(1):16-23.

38. Chapados R, Abe K, Ihida-Stansbury K, McKean D, Gates AT, Kern M, et al. ROCK controls matrix synthesis in vascular smooth muscle cells: coupling vasoconstriction to vascular remodeling. Circ Res. 2006;99(8):837-44.

39. Schellings MW, Pinto YM, Heymans S. Matricellular proteins in the heart: possible role during stress and remodeling. Cardiovasc Res. 2004;64(1):24-31.

40. Satta J, Melkko J, Pollanen R, Tuukkanen J, Paakko P, Ohtonen P, et al. Progression of human aortic valve stenosis is associated with tenascin- $C$ expression. J Am Coll Cardiol. 2002;39(1):96-101.

41. Ishiyama Y, Gallagher PE, Averill DB, Tallant EA, Brosnihan KB, Ferrario CM. Upregulation of angiotensin-converting enzyme 2 after myocardial infarction by blockade of angiotensin II receptors. Hypertension (Dallas, Tex: 1979). 2004;43(5):970-6.

42. Santer D, Nagel F, Goncalves IF, Kaun C, Wojta J, Fagyas M, et al. Tenascin-C aggravates ventricular dilatation and angiotensin-converting enzyme activity after myocardial infarction in mice. ESC Heart Fail. 2020;7(5):2113-22.

43. Rajamannan NM, Evans FJ, Aikawa E, Grande-Allen KJ, Demer LL, Heistad $\mathrm{DD}$, et al. Calcific aortic valve disease: not simply a degenerative process: a review and agenda for research from the National Heart and Lung and Blood Institute Aortic Stenosis Working Group. Executive summary: Calcific aortic valve disease-2011 update. Circulation. 2011;124(16):1783-91.

44. Chen JH, Yip CY, Sone ED, Simmons CA. Identification and characterization of aortic valve mesenchymal progenitor cells with robust osteogenic calcification potential. Am J Pathol. 2009;174(3):1109-19.

45. Shirakawa K, Endo J, Kataoka M, Katsumata Y, Anzai A, Moriyama H, et al. MerTK expression and ERK activation are essential for the functional maturation of osteopontin-producing reparative macrophages after myocardial infarction. J Am Heart Assoc. 2020;31:e017071.

46. Matilla L, Roncal C, Ibarrola J, Arrieta V, Garcia-Pena A, Fernandez-Celis A, et al. A role for MMP-10 (Matrix Metalloproteinase-10) in calcific aortic valve stenosis. Arterioscler Thromb Vasc Biol. 2020;40(5):1370-82.

47. Monzack EL, Masters KS. Can valvular interstitial cells become true osteoblasts? A side-by-side comparison. J Heart Valve Dis. 2011;20(4):449-63.

48. Laguna-Fernandez A, Carracedo M, Jeanson G, Nagy E, Eriksson P, Caligiur $G$, et al. Iron alters valvular interstitial cell function and is associated with calcification in aortic stenosis. Eur Heart J. 2016;37(47):3532-5.

49. Rittling SR. Osteopontin in macrophage function. Expert Rev Mol Med. 2011;26(13):e15.

50. Vadana M, Cecoltan S, Ciortan L, Macarie RD, Tucureanu MM, Mihaila AC, et al. Molecular mechanisms involved in high glucose-induced valve calcification in a 3D valve model with human valvular cells. J Cell Mol Med. 2020;24(11):6350-61.

51. Kossar AP, Anselmo W, Grau JB, Liu Y, Small A, Carter SL, et al. Circulating and tissue matricellular RNA and protein expression in calcific aortic valve disease. Physiol Genomics. 2020:52(4):191-9.

52. Bartolomucci A, Possenti R, Mahata SK, Fischer-Colbrie R, Loh YP, Salton SR. The extended granin family: structure, function, and biomedical implications. Endocr Rev. 2011;32(6):755-97.

53. Fischer-Colbrie R, Laslop A, Kirchmair R. Secretogranin II: molecular properties, regulation of biosynthesis and processing to the neuropeptide secretoneurin. Prog Neurobiol. 1995;46(1):49-70.

54. Helle KB. Regulatory peptides from chromogranin A and secretogranin II: putative modulators of cells and tissues involved in inflammatory conditions. Regul Pept. 2010;165(1):45-51.

55. Fischer-Colbrie R, Kirchmair R, Kähler CM, Wiedermann CJ, Saria A. Secretoneurin: a new player in angiogenesis and chemotaxis linking nerves, blood vessels and the immune system. Curr Protein Pept Sci. 2005;6(4):373-85

56. Yan S, Wang $X$, Chai H, Wang H, Yao Q, Chen C. Secretoneurin increases monolayer permeability in human coronary artery endothelial cells. Surgery. 2006;140(2):243-51.

57. Kähler CM, Fischer-Colbrie R. Secretoneurin-a novel link between the nervous and the immune system. Conservation of the sequence and functional aspects. Adv Exp Med Biol. 2000;482:279-90.

58. Albrecht-Schgoer K, Schgoer W, Holfeld J, Theurl M, Wiedemann D, Steger $C$, et al. The angiogenic factor secretoneurin induces coronary angiogenesis in a model of myocardial infarction by stimulation of vascular endothelial growth factor signaling in endothelial cells. Circulation. 2012;126(21):2491-501.

59. Liu W, Wang F, Zhao M, Fan Y, Cai W, Luo M. The neuropeptide secretoneurin exerts a direct effect on arteriogenesis in vivo and in vitro. Anat Rec (Hoboken, NJ: 2007). 2018;301(11):1917-27.

60. Weiss RM, Miller JD, Heistad DD. Fibrocalcific aortic valve disease: opportunity to understand disease mechanisms using mouse models. Circ Res. 2013;113(2):209-22.

61. Zhang H, Zhu Q, Cui J, Wang Y, Chen MJ, Guo X, et al. Structure and evolution of the Fam20 kinases. Nat Commun. 2018;9(1):1218.

62. Nalbant D, Youn H, Nalbant SI, Sharma S, Cobos E, Beale EG, et al. FAM20: an evolutionarily conserved family of secreted proteins expressed in hematopoietic cells. BMC Genomics. 2005;27(6):11.

63. O'Sullivan J, Bitu CC, Daly SB, Urquhart JE, Barron MJ, Bhaskar SS, et al. Whole-Exome sequencing identifies FAM20A mutations as a cause of amelogenesis imperfecta and gingival hyperplasia syndrome. Am J Hum Genet. 2011;88(5):616-20. 
64. Jaureguiberry G, De la Dure-Molla M, Parry D, Quentric M, Himmerkus N, Koike T, et al. Nephrocalcinosis (enamel renal syndrome) caused by autosomal recessive FAM20A mutations. Nephron Physiol. 2012;122(1-2):1-6.

65. Zhao Y, Su H, Shen X, Du J, Zhang X, Zhao Y. The immunological function of CD52 and its targeting in organ transplantation. Inflamm Res. 2017;66(7):571-8.

66. Krishnaswamy JK, Alsen S, Yrlid U, Eisenbarth SC, Williams A. Determination of T follicular helper cell fate by dendritic cells. Front Immunol. 2018:9:2169.

67. Rashidi M, Bandala-Sanchez E, Lawlor KE, Zhang Y, Neale AM, Vijayaraj SL, et al. CD52 inhibits Toll-like receptor activation of NF-kappaB and triggers apoptosis to suppress inflammation. Cell Death Differ. 2018;25(2):392-405.

68. Summerhill VI, Moschetta D, Orekhov AN, Poggio P, Myasoedova VA. Sex-specific features of calcific aortic valve disease. Int J Mol Sci. 2020;21(16):5620.

\section{Publisher's Note}

Springer Nature remains neutral with regard to jurisdictional claims in published maps and institutional affiliations.
Ready to submit your research? Choose BMC and benefit from:

- fast, convenient online submission

- thorough peer review by experienced researchers in your field

- rapid publication on acceptance

- support for research data, including large and complex data types

- gold Open Access which fosters wider collaboration and increased citations

- maximum visibility for your research: over $100 \mathrm{M}$ website views per year

At BMC, research is always in progress.

Learn more biomedcentral.com/submissions 